Brit. J. industr. Med., 1956, 13, 55.

\title{
DERMATITIS FROM MANSONIA WOOD
}

\author{
BY \\ L. B. BOURNE \\ From Cossor House, London
}

(RECEIVED FOR PUBLICATION JUNE 16, 1955)

Horner and Wigley (1936) reported a case of dermatitis due to Mansonia wood (Sterculiacea altissima) and in his annual report the Chief Inspector of Factories (1936) also reported that the dust of this wood could give rise to serious symptoms and in this respect compared it with African box wood.

There are apparently no other reports of the possible dangers relating to the use of this wood, and, as it has been imported in recent years in much larger quantities than hitherto, an account of an outbreak of dermatitis is likely to be of interest as other outbreaks are known to have occurred elsewhere in this country. The wood itself is exported from the Gold Coast and Nigeria and is used locally for general carpentry and in railway coaches. As it resembles black walnut it has been used for piano cases, furniture, shop fittings, and camera boxes. It has been found very useful as a substitute for true walnut with which it should not be confused. In the fresh state the wood is dark brown but on exposure changes to light brown. It air-seasons readily and has considerable natural durability. It was recently found to be ideal for use in the manufacture of radio and television cabinets and the outbreak reported here took place in a factory which makes these products.

All the men affected worked in one small department although the wood in its various stages through the factory was handled by many other workers. On investigation the men in the department concerned were those engaged in fine sanding, whereas in the rest of the factory, although the same wood was handled by many other workers, no men appeared to be involved.

The wood enters the factory in planks which may vary in colour from light brown to a darkish grey brown, the bark remaining much paler. It is then rough sawn and planed in the mill and the particles of reasonably large size are thrown off and extracted by an efficient exhaust system. The work of assembly into cabinets also appears to carry no risk as at no time have there been complaints. It is only when the cabinets are taken to the fine sanding department that difficulties arise. Here the wood is treated by hand sanding with fine carborundum type papers to smooth the surface for further treatment with stains and lacquers. This department has an efficient ventilation system proved to be satisfactory by years of experience with wood dusts of all types. The fine powder given off during the sand papering operation has been shown to be the direct cause of the symptoms.

Twelve men worked in the department, many of whom had been there for some years using many types of wood, but this was the first occasion on which any of them had complained. Six of the men were markedly affected and one only slightly.

The first symptoms were those of nasal irritation and sneezing followed by itching which increased during the working week but diminished considerably during the week-end. If prolonged contact with the dust was maintained there followed increased irritation which spread from the exposed areas of the skin. This was followed by sores in the nostrils, discharge from the nose and epistaxis, and in some cases discharge from the external auditory meatus. Nausea was not marked and there were no complaints of weakness of the arms or legs as previously described by Horner and Wigley (1936).

The dermatitis is characterized by an erythema which is followed by a folliculitis with exudation and some exfoliation. Occasionally there is considerable oedema of the face particularly in the orbital regions (Fig. 1). The fine powder settles on the head and, when the hair is combed, gives rise to an irritation of the scalp, the neck, and between the shoulder blades. It also may settle inside the clothing anywhere but does so particularly in the socks (Fig. 2). Sore throats are common.

It was interesting to note that during the annual holiday period all those involved improved very much and when they returned to work after two weeks' rest they were practically symptom free. There was an immediate recurrence once the work 


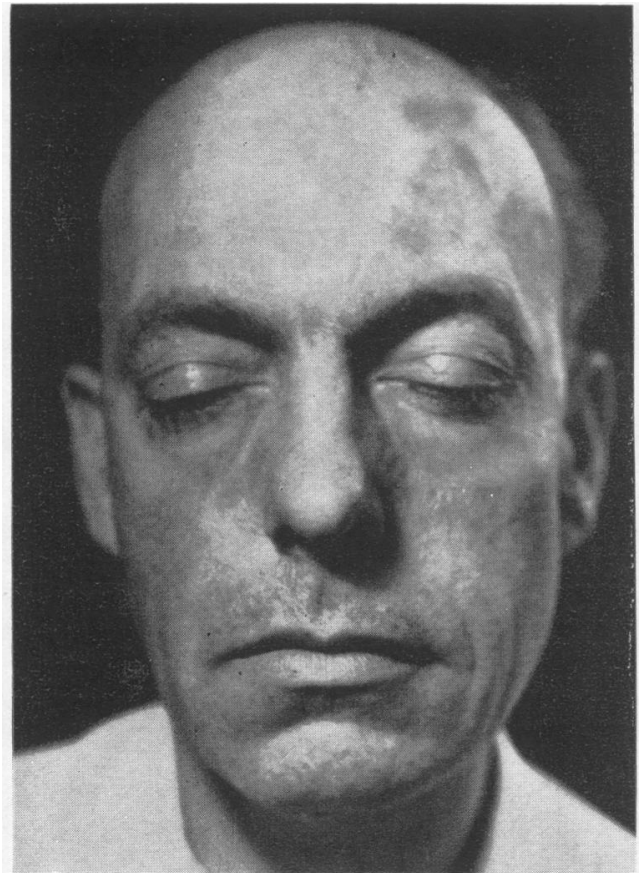

Fig. 1.-Case F. H. Erythema of face and forehead. Periorbital oedema and ulceration of left eyelid.

was restarted. Protective masks (Martindale type) and barrier creams were supplied but although these did reduce the nasal symptoms and protect the hands, the rest of the exposed parts of the face, ears, and scalp continued to be affected. Patch tests of six workers gave a marked result in eight to 24 hours with an erythematous itching area which persisted for as long as four days, becoming indurated at the end of this period and gradually fading after some weeks.

The method of testing was as follows :-

An area about 2 in. square of both forearms on the flexor surface was cleaned with ether and allowed to dry. Some Mansonia dust was put on sterile gauze and this was applied to one arm and covered with sticking plaster. This procedure was repeated on the other arm without any dust. A further control was carried out on the author using a similar procedure. Local irritation was felt in about 12 hours and a marked erythema in 24 hours ; the offending powder was then removed. The powder appeared to be unchanged but the skin affected was erythematous, slightly raised, and had to be treated with antipruritics for about 10 days.

It was decided to stop using the wood and all those workers who have remained in the employ of the factory have recovered, are symptom free and

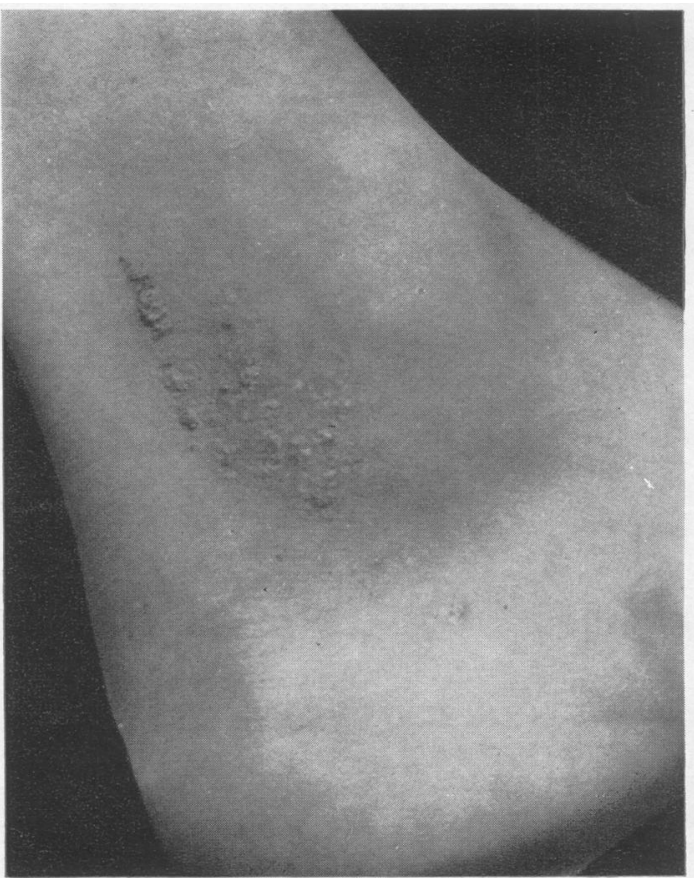

Fig. 2.-Same case. Typical dermatitis from dust inside sock.

are not affected by the other woods in general use, the majority being imported, some of tropical origin.

Two workers left the employ of the company and no further records are available.

\section{Case Histories}

Case 1.-F. H., aged 31, a woodworker, began work at the factory on January 2, 1950. On March 5, 1953, he complained of itching fingers after using "special" wood for some days but had no obvious signs. The itching subsided at the week-end but began again within a few hours of using the wood. He did not again use the wood until July 20 and on July 23 had itching fingers with erythema of the backs of the hands and forearms. On August 10, 1953, he returned to work after a holiday with a clear skin. On August 12 he complained of irritation of the nose and itching hands. By the next day the irritation of the hands and forearms had increased, he had a discharge from the ears, superficial ulceration of the nostrils, and marked erythema of the face and scalp. He was taken off Mansonia wood.

On August 21 a patch test of the arms with Mansonia wood powder gave a marked reaction in 12 hours. By August 25 the patch area-indurated, erythematous, tingling - was still marked but the involved areas of the face, arms, and nose were clearing. By December 15 the skin was clear and the other symptoms had subsided. 
On May 3, 1954, he used Mansonia wood for a small job and within a few minutes tingling began.

Case 2.-H. P., aged 47, a woodworker, began work on September 27, 1948. From March 9 to 16, 1953, he was absent with a certificate of " acute coryza" after using the wood for some days. On April 20 he had an itching erythema on both arms and face after a few days' exposure. On April 22 a patch test gave a marked positive reaction in 24 hours, and the patient was taken off the work. He has had no trouble since.

Case 3.-J. W., aged 34, a woodworker, began work on March 18, 1953. On May 27 he had an irritation on the left shin, which he stated had been there for some time, clearing up and recurring. It cleared up with lin. cal.

On August 18 the leg was clear but the patient now complained of irritation of the face and between the fingers. He also had oedema and erythema of the upper lip and cheeks, the periocular areas, and the brow. He was taken off the work and the skin cleared. On April 22 a patch test had given a marked positive reaction in 24 hours.

Case 4.-D. A., aged 33, a woodworker, worked at this factory from September 29, 1948, to January 3, 1952, and then again from January 9, 1953. On October 5 he had a cold with rhinitis which persisted and erythema of the hands and face which was not reported. He was off work. On October 27 he reported with dermatitis of the ears, neck, ankles, and axillae. On October 26 a patch test had given a marked positive reaction in eight hours. By the morning of December 15 the skin was clear. In the afternoon of that day he was further exposed to dust for half an hour, and the erythema and pruritus recurred. On March 24 he was exposed to Mansonia wood in error and symptoms returned.

Case 5.-H. Y., aged 47, a woodworker, began work in February, 1953. On October 7 he started to use Mansonia wood and in two days had an irritation of the face, arms, and hands. This was relieved overnight but began again next morning at work. This sequence continued for two weeks. On October 23 he reported to the surgery with follicular erythema of both arms and exudative dermatitis of the cubital fossae, erythema of the face, and follicular dermatitis on the centre of the back between the scapulae. A patch test on October 26 gave a marked positive reaction in 12 hours. By December 15 the skin was clear.

No worker gave a history of previous skin involvement and all had been examined for skin disease before starting work. All the workers had previous experience with many woods for periods varying from 14 to 33 years. The dates of the first exposure to the wood vary because after the first outbreak in March, 1953, the work was re-routed through the factory at different times in order to check the effect of the wood on different operatives. The department in which fine sanding was carried out was separate and did not form part of the rest of the factory, and the incidence of colds and upper respiratory tract affections was no higher there than in any other part of the factory in previous years.

\section{Discussion}

The Chief Inspector's report quoted states that " an analysis on Mansonia wood is being done and it is possible that an alkaloid similar to that present in African box wood will be found ".

The Officer in Charge, Chemistry Section of The Forest Products Research Laboratory, states :

"We have had other reports that the fine dust produced in working this timber is liable to cause irritation to the eyes, nose, and throat and there have been some cases of dermatitis attributed to this cause. . . . Information on the chemical components of woods responsible for their toxic or irritant effects is very scanty. In the case of Mansonia nothing appears to be known about the chemical nature of the minor components of the wood, and I do not think it can be assumed that the offending substance is an alkaloid unless direct evidence is available on this point. We have investigated one or two timbers here with the object of obtaining information on the types of component which produce these toxic effects, but with rather indefinite results. Thus the Trinidad timber toporite (Hernandia sonora), which causes skin irritation, contains the acetate of podophyllotoxin, which might be expected to be the responsible component, but the medical tests we had carried out were inconclusive.

"In a recent paper Sandermann and Barghoorn report that lapachonone isolated from the South American timber peroba de campos (Paratecoma peroba) has irritant properties, although it had been stated earlier that the toxicity of this wood was due to resin acids in it. I mention these examples merely to emphasize the discrepancies and the lack of wellestablished data which prevail in this field. You will notice that neither podophyllotoxin nor lapachonone is an alkaloid.

"The chemical examination of wood components is rather a lengthy process. It is necessary to extract the wood with one or more solvents, e.g., water, alcohol, ether, fractionate the extracts further by some suitable method, and examine the various fractions for their physiological effects. If they produce positive effects they must be further purified, for they are generally complex mixtures, and characterized chemically. I do not think there is any effective short cut to this kind of investigation."

Schwartz, Tulipan, and Peck (1947) state :

"Wood from foreign localities or woods not handled before are the ones that usually cause dermatitis".

The workers become affected several days after beginning to work with the new wood. It is usually a sensitization dermatitis, only a few of the workers being affected. The majority of those affected become "hardened" and develop a hyposensitization after they recover. A small percentage of those affected remain sensitized. 
In spite of the fact that the wood was excellent for the purpose and economical in use, the decision to withdraw the material was made because the risk of the loss of skilled personnel due to absence from work and also from those who feared contact was too great. Fine sanding is a skilled operation and workers must have long experience. The suggestion that " hardening " against the offending material is possible was also considered, but our experience with chemicals is such that increased sensitization oftens takes place and that the hyposensitization after recovery, as mentioned by Schwartz and others, appears to be rare amongst those who react strongly to exogenous irritants.

Since the first outbreak in 1953 other woods have been in daily use. In view of our previous experience over many years, particularly with hard woods of tropical origin, none of which have been troublesome, it was felt that further patch tests with these woods were not as effective as the continual handling and exposure to the fine dust, deposited on the skin and other areas which have been affected by Mansonia dust. Ordinary washing facilities have proved adequate and barrier creams have been used as a routine in the department for many years.

Clinically, the interest is directed towards the complaint of the patient that he "caught a cold in the nose ", as the irritant dust may rise and affect the nasal mucous membrane which becomes oedematous. This was noted in at least three cases where the hands of these patients were of the hyperkeratotic type and would almost certainly have offered a greater resistance to the offending dust and, as a result, the pruritus of the hands appears to have been delayed for a few days.

\section{Summary}

The outbreak of toxic symptoms from the use of Mansonia wood (Sterculiacea altissima) is described in which seven out of 12 men engaged on fine sanding were involved. Other cases have been known in the industry. The commonest symptoms were rhinitis, dermatitis, and oedema. It had been previously reported that collapse may occur but was not the case in any of the workers under observation. Information as to the irritant responsible is not available.

\section{REFERENCES}

Chief Inspector of Factories (1936). Annual Report for 1935, p. 60. Dept. of Scientific and Indust. Res., Forest Prod. Res. Lab. Office in Charge, Chemistry Section (personal communication).
Horner, S., and Wigley, J. E. M. (1936). Brit. J. Derm., 48, 26. Sandermann, - -, and Barghoorn, - - (1955). Holzforschung, 9, 112 .

Schwartz, L., Tulipan, L., and Peck, S. M. (1947). Occupational Diseases of the Skin, 2nd ed. p. 590. Lea and Febiger, Philadelphia. 